\title{
Star Forming Objects in the Tidal Tails of Compact Groups
}

\author{
J. Iglesias-Páramo \\ jiglesia@ll.iac.es \\ Instituto de Astrofísica de Canarias \\ 38200 La Laguna, Tenerife, SPAIN \\ and \\ J.M. Vílchez \\ jvm@iaa.es \\ Instituto de Astrofísica de Andalucía (CSIC) \\ Apdo. 3004, 18080 Granada, SPAIN
}

Received __; accepted _ 


\begin{abstract}
A search for star forming objects belonging to tidal tails has been carried out in a sample of deep $\mathrm{H} \alpha$ images of 16 compact groups of galaxies. A total of 36 objects with $\mathrm{H} \alpha$ luminosity larger than $10^{38} \mathrm{erg} \mathrm{s}^{-1}$ have been detected in five groups. The fraction of the total $\mathrm{H} \alpha$ luminosity of their respective parent galaxies shown by the tidal objects is always below $5 \%$ except for the tidal features of HCG 95, whose $\mathrm{H} \alpha$ luminosity amounts to $65 \%$ of the total luminosity. Out of this 36 objects, 9 star forming tidal dwarf galaxy candidates have been finally identified on the basis of their projected distances to the nuclei of the parent galaxies and their total $\mathrm{H} \alpha$ luminosities. Overall, the observed properties of the candidates resemble those previously reported for the so-called tidal dwarf galaxies.
\end{abstract}

Subject headings: Galaxies: evolution — starburst — interactions; ISM: HII regions 


\section{Introduction}

Interactions between disk galaxies are known to produce strong morphological disturbances and, if the geometry of the encounter is favorable, to enhance the star formation rates of the disk galaxies. The pioneering work by Tomre \& Tomre (1972) and a later study by Barnes (1988) supported the original idea by Zwicky (1956) that dwarf galaxies could originate within the tidal tails resulting from a strong interaction between two disk galaxies. Since then, several examples of dwarf galaxies with tidal origin have already been detected in interacting systems in an advanced stage of merging and also around galaxies experiencing less dramatic interactions (Schweizer 1978, Mirabel et al. 1991,1992, Hibbard \& van Gorkom 1996, Duc \& Mirabel 1997,1998).

The compact group environment has been suggested to be an ideal place for interactions between galaxies given their large spatial densities and their low velocity dispersions. Mendes de Oliveira \& Hickson (1994) reported morphological signs of interaction in many galaxies of compact groups from Hickson's Catalogue (1982). Other recent studies on the photometric properties of the galaxies in Hickson Compact Groups at different wavelengths resulted contradictory when compared to samples of field galaxies: while $\mathrm{CO}, \mathrm{FIR}, \mathrm{H} \alpha$ and optical fluxes seem to be similar to those measured for field galaxies (Moles et al. 1994, Verdes-Montenegro et al. 1998, Iglesias-Páramo \& Vílchez 1999), radio continuum and $21 \mathrm{~cm}$ emission are on average lower than for samples of isolated galaxies (Williams \& Rood 1987, Menon 1995, Huchtmeier 1997).

Hunsberger et al. (1998) carried out a search for dwarf galaxy candidates in the close environment of a sample of Hickson Compact Groups, by automated detection of faint objects on deep $R$ band

frames. After decontamination for the presence of background galaxies, they found that the luminosity function in compact groups showed an enhancement at the faint luminosity end compared to a normal Schechter function. They concluded that the initial dwarf galaxy population in compact groups is replenished by "subsequent generations" formed in the tidal debris of giant galaxy interactions.

In this work we present the results of a search for tidal star forming dwarfs in our $\mathrm{H} \alpha$ images of a sample of nearby compact groups (see Vílchez \& Iglesias-Páramo 1998, Iglesias-Páramo \& Vílchez 1999). Five of the groups were found to show optically prominent tidal tails with star forming objects within them. We emphasize the fact that all the detected objects are $\mathrm{H} \alpha$ emitters, therefore implying that they are physically associated with the galaxies of the group. The main properties of the star forming objects will be presented in $\oint_{2}$, as well as a description of their close environment. $\S 3$ contains a discussion on their likely evolutionary scenario.

\section{The Sample}

Deep $\mathrm{H} \alpha$ images for a total of 67 galaxies belonging to 16 Hickson compact groups were inspected. Four telescopes were used in order to obtain all the data presented in this paper: The INT $2.5 \mathrm{~m}$ and JKT 1.0m in the Observatorio del Roque de los Muchachos, the IAC80 in the Observatorio del Teide and the 2.2m Telescope in the Observatorio Hispano-Alemán de Calar Alto. Also, some $R$ band continuum frames taken at the $3.6 \mathrm{~m}$ CFHT were kindly made available to us by Dr. Paul 
Hickson. More details concerning the data acquisition and reduction for the sample can be found in Iglesias-Páramo \& Vílchez (1999)円. H $\alpha$ emitting objects in tidal features were found in 5 groups of the sample: HCG 31, HCG 44, HCG 92, HCG 93 and HCG 95. We note that although tidal features were reported for some other groups of our sample (Mendes de Oliveira \& Hickson 1994), no H $\alpha$ objects have been found associated with these features.

Figures 1 to 5 show detailed contour maps of continuum subtracted $\mathrm{H} \alpha$ images of the sections of the groups where star forming tidal objects were detected. The contour scales are different for each group. A preselection of star forming objects was done by careful optical inspection of the $\mathrm{H} \alpha$ images, with the following criteria: (1) all the objects appear spatially resolved at the $3 \sigma$ isophote over the sky level, (2) they are more luminous than $10^{38} \mathrm{erg} \mathrm{s}^{-1}$ - this somewhat arbitrary luminosity limit was chosen in order to exclude normal low luminosity HıI regions (see $\S 3$ ) - (3) they are located along tidal features, well away from the main body of the parent galaxies.

Table 1 shows some physical properties of the tidal objects. Column 1 shows the identifier of each object as quoted in Figures 1 to 5 (with the prefix TD added to the identifier); Column 2 shows the name of the parent galaxy according to Hickson et al. (1989); Column 3 shows the optical radius at the $25 \mathrm{mag}$ arcsec $^{-2}$ isophote of the parent galaxy, $R_{25}$; Column 4 shows the measured H $\alpha$ luminosity in erg s${ }^{-1}$; Column 5 shows the fraction of the total $\mathrm{H} \alpha$ luminosity emitted by the tidal objects; Column 6 shows the $\mathrm{H} \alpha$ equivalent width in $\AA$; Column 7 shows the projected distance of the tidal object to the center of the parent galaxies normalized to $R_{25}$. All the distance-dependent quantities have been estimated adopting $H_{0}=75 \mathrm{~km} \mathrm{~s}^{-1} \mathrm{Mpc}^{-1}$ and assuming a pure Hubble flow?. Here follows a brief morphological description of the close environment of the detected tidal objects.

\subsection{HCG 31}

This group, that hosts up to nine tidal objects, has been previously discussed in the literature (i.e. Rubin et al. 1990, Iglesias-Páramo \& Vílchez 1997a, Johnson et al. 1999). The parent galaxies seem to be in an advanced stage of merging, and show strong star formation activity everywhere. A third galaxy located to the West could be joining the interacting pair. The whole group is immersed in an Hi cloud (Williams et al. 1991) and is one of the few Hickson Compact Groups richer than expected in HI. The tidal tail containing objects TD 31c to TD 31i, appears also delineated in the Hi map. The large equivalent widths measured for some of the tidal objects, together with their extremely blue broad band colors (Iglesias-Páramo \& Vílchez 1997a), suggest that we are probably observing two recently originated dwarf galaxies. This is remarkable since this kind of objects were thought to be born mostly in systems containing early-type galaxies (Duc \& Mirabel 1998) without apparent optical spiral arms.

\footnotetext{
${ }^{1}$ Although not mentioned in that paper, HCG 80 belongs to the current sample

${ }^{2}$ Note that the $\mathrm{H} \alpha$ luminosities published in our previous papers adopted a different value for $H_{0}$
} 


\section{2. $\mathrm{HCG} 44$}

HCG 44 is quite a dispersed group. Overall, it was found to be deficient in Hi (Williams \& Rood 1987). The individual galaxies HCG 44a and c were also found to be Hi deficient. An Hi dwarf galaxy was detected within the field of the group at approximately the same radial velocity (Williams et al. 1991). The parent galaxy hosting the tidal objects, HCG 44d, is a SB(s)c peculiar galaxy showing two distorted tidal arms perpendicular to the direction of the central bar (Mendes de Oliveira \& Hickson 1994). Almost no underlying emission from the tidal objects was detected in broad band images. The HI map of this galaxy also shows asymmetries and clear signatures of interactions (Williams et al. 1991). The nearby galaxy HCG 44a, an early-type spiral with the plane of the disk perpendicular to the tidal tails of HCG 44d, could be responsible for the interaction suffered by HCG 44d; the distortion detected in the outer disk of HCG 44a towards HCG 44d is a relic of the interaction between both galaxies. Eight star forming objects were detected in the tidal tails of HCG 44d.

\subsection{HCG 92}

Several tidal objects were detected in this group, widely known as Stephan's Quintet. Interesting reviews of the history and evolution of this group can be found in Moles et al. $(1997,1998)$ and Xu et al. (1999). The Hi distribution is decoupled from the optical galaxies (Verdes-Montenegro et al., 2000), which could explain the low star formation activity of this group in spite of the high rate of interaction among its members. One of the tidal objects, TD 92a, is located at the tip of a long tidal tail extending out from HCG 92c. This galaxy shows a Seyfert 2 type active nucleus, a strong bar across the disk and several shells apparent in the continuum image. However, excepting the nucleus, no strong $\mathrm{H} \alpha$ emission was detected throughout this galaxy. Xu et al. (1999) performed a detailed study of the star forming regions in HCG 92 based on IR imaging, and reported this tidal dwarf galaxy as probably due to the interaction of HCG 92c with the nearby galaxy NGC 7320c, not included as a member of the group in the original catalog. The rest of the tidal objects were detected around the interacting pair of galaxies HCG 92b and d. Each of these galaxies has developed one tidal tail pointing towards the North. Our H $\alpha$ map of the tail associated with HCG 92b shows a vertical bar-like structure, probably containing shocked gas (Ohyama et al. 1998) due to the intrusion of this galaxy into the group. At the tip of this tail, a starburst region was reported by Xu et al. (1999) - our object TD 92b - and attributed to the collision of the galaxy with the intergalactic medium of the group. We have detected several other objects in the outskirts of HCG 92d.

\subsection{HCG 93}

Only one galaxy, HCG 93b, hosts the seven tidal objects detected in this group. HCG 93 is a very Hi poor compact group. The mean separation between its galaxies is about $70 k p c$, which makes it the largest of the five groups reported in this work. The parent galaxy, HCG 93b, is an SB(s)cd peculiar galaxy with two long tails, well visible in the optical continuum images. This galaxy, together with the massive elliptical HCG 93a was cataloged as a binary system by Peterson (1979). The low 
surface brightness asymmetric halo surrounding HCG 93a (see the red continuum image in Vílchez \& Iglesias-Páramo 1998) suggests that an interaction between the two galaxies may be responsible for the tidal tails exhibited by HCG 93b as well as for the faint halo of HCG 93a. Four of the tidal objects - including the most luminous one - were detected along the Northern tail, and the remaining three along the Southern one.

\subsection{HCG 95}

In this group of galaxies, a three-galaxy interaction seems to be taking place (Iglesias-Páramo \& Vílchez 1998). As a result, four tidal tails emerge from the doubly nucleated galaxy HCG 95c. The gas distribution of the system appears peculiar because it seems weighted towards the Eastern nucleus of HCG 95c and the Northern tidal tail emerging from it, with almost no gas in the rest of the system.

Four tidal objects were detected along the Northern gas-rich tail. A close inspection of the broad band and $\mathrm{H} \alpha$ images of the system suggested the possibility that transfer of material was taking place from the most luminous object in this tidal tail - namely TD 95b - towards the nucleus of the bright nearby elliptical galaxy HCG 95a (Iglesias-Páramo \& Vílchez 1997b). The analysis of the broad band colors of the tidal objects showed that the amount of underlying population present along the Northern tidal tail probably decreases outwards, being almost negligible for TD 95a and TD 95b.

\section{Discussion and Conclusions}

As can be seen from Table 1, the $\mathrm{H} \alpha$ equivalent widths and luminosities of the tidal objects suggest that most of them host very young star formation bursts and are above the luminosity limit for giant His regions. The underlying continuum luminosity varies greatly from object to object as shown in Figures 1 to 5. This is the reason for the large range covered by their $\mathrm{H} \alpha$ equivalent width values, rather than the result of different evolutionary stages of the corresponding star formation bursts of the tidal objects. The fraction of the total $\mathrm{H} \alpha$ flux corresponding to tidal objects is typically under $5 \%$ (see Table 1), except in the remarkable case of the tidal features in HCG 95c, which amount to $65 \%$ of the total luminosity of the galaxy. We attribute this extraordinarily high fraction of luminosity within the tidal tail to the fact that HCG 95c belongs to a triple system showing a rather complicated interaction sequence (Iglesias-Páramo \& Vílchez 1998).

The existence of these tidal objects is linked to the possibility that they could subsequently evolve as independent dwarf galaxies, like those observed at the tips of the tidal tails of the Antennae and the Superantennae (Mirabel et al. 1991,1992). Looking to the optical images, only rough morphological similarities between the tails of the Antennae and Superantennae and the tails shown by our galaxies can be found. The Antennae and Superantennae fall in the most dramatic case of interaction of a pair of galaxies, whereas our five groups span a wide range of interaction strengths. At this point, two critical questions arise concerning the possibility that tidal objects can evolve independently as dwarf galaxies: (1) are they distant enough from the parent galaxy so as to be able to escape from the potential well?, and (2) are they massive enough to be self gravitating systems and to be considered 
dwarf galaxies?

Concerning the first question, some authors have claimed (Schweizer 1978; Hibbard \& van Gorkom 1996) that most of the material ejected during an interaction is accumulated in the tidal tails. N-body simulations suggest that a substantial fraction of this material will slowly fall into the main body of the remnant of the interaction, and only the outermost $20 \%$ will probably gain enough kinetic energy to be able to evolve independently for a long time (see Hibbard \& Mihos 1995). Given that no information is available on the velocity fields of the interacting pairs of our sample, and that they show a great variety of interaction patterns, we will adopt a quantitative criterion based on the projected distances of the tidal objects from the nuclei of their parent galaxies in order to select the most likely candidates to escape from the potential well. Ferguson et al. (1998) carried out a deep search for HiI regions well outside the optical limits of a sample of disk galaxies, and found no HiI regions located at a projected galactocentric distance larger than $2 \times R_{25}$. This result was explained as due to the fact that the gas surface density is below the critical density for star formation at such large radii from the centers of the galaxies. Therefore, we can consider that those star forming regions located that far from the nuclei of their parent galaxies must have been originated by an external mechanism which has removed gas from the inner regions of the parent galaxies. Star forming regions far away from their parent galaxies may become kinematically decoupled from the stellar disks; thus they are very likely escaping the potential well of the parent galaxy and could become good tidal dwarf galaxy candidates. According to Table 1, only 9 tidal objects fulfill this criterion $\left(R>2 \times R_{25}\right)$. Obviously, this is a conservative criterion adopted given the lack of information on the velocity field of the tidal tails. Therefore, we should bear in mind that because of possible projection effects, the number of tidal dwarfs candidates selected is a lower limit since other objects not satisfying this criterion cannot be ruled out to be tidal dwarf galaxies.

Concerning the second question posed above, Elmegreen et al. (1993) proposed that clouds as massive as $10^{8} M_{\odot}$ can form during interactions of disk galaxies. Under some conditions, these clouds could become dwarf galaxies. In particular, when the mass of the perturber is larger than approximately 1.4 times the mass of the parent galaxy, - as appears to be the case for the five groups of our sample presenting $\mathrm{H} \alpha$ emitting tidal tails - these models predict that the outermost clouds are very likely to escape the potential well. Such $10^{8} M_{\odot}$ clouds would produce $10^{6} M_{\odot}$ starbursts, assuming a value for the efficiency of star formation of $1 \%$ (see Kennicutt 1998 and references therein). From the observational side, the $\mathrm{H} \alpha$ luminosities reported for the tidal dwarf galaxies of the Antennae (Mirabel et al. 1991) and NGC 5291 (Duc \& Mirabel 1998), are comparable to the ones derived in the present paper; the Hi masses measured for those dwarfs appear to be typically above $10^{8} M_{\odot}$. In the same direction, from a large compilation of dwarf galaxies Hunter \& Gallagher (1986) reported HI masses larger than $10^{8} M_{\odot}$ for objects with $L_{\mathrm{H} \alpha} \gtrsim 10^{39} \mathrm{erg} \mathrm{s}{ }^{-1}$. Such bright objects are known to be gravitationally bound, according to the well established $L(\mathrm{H} \alpha)$ versus $\sigma$ relationship for HiI galaxies (Terlevich \& Melnick 1981). The 9 objects selected above show $\mathrm{H} \alpha$ luminosities larger than $10^{39} \mathrm{erg} \mathrm{s} \mathrm{s}^{-1}$, which on observational grounds seems to be a reasonable lower limit to ensure self

\footnotetext{
${ }^{3}$ estimated from a comparison of the magnitudes of the parent galaxies with those of the remaining galaxies in the groups
} 
gravitation for His complexes.

The two restrictions imposed above, though conservative, appear reasonable since HiI regions brighter than $10^{39} \mathrm{erg} \mathrm{s}^{-1}$ are found to be located at galactocentric distances lower than about $1.2 \times R_{25}$ (as derived from the HiI region surveys by Rozas et al. 1996,2000). A naive estimation of the dynamical time needed by our dwarf galaxy candidates to travel a distance equivalent to $R_{25}$ as a consequence of the interaction would be of the order of the fading time for His regions. Thus, we are confident that the 9 selected candidates must have been generated during the interaction and do not belong to a previous generation of disk HiI regions.

From the arguments presented above, it appears clear that the 9 star forming objects finally selected represent good candidates to satisfy the escape and self gravitation conditions, and thus they can be included in the group of the so-called tidal dwarf galaxies. Further information on total masses and relative velocity with respect to their parent galaxies is required to confirm that they satisfy both conditions.

It seems striking that the ratio between the number of candidate dwarf galaxies to the total number of tidal objects detected, 25\%, appears consistent with the fraction of the ejected material which is expected to escape according to theoretical models (Hibbard \& van Gorkom 1995). Since all these candidate tidal galaxies were selected at projected distances larger than $2 \times R_{25}$, we suggest that this last criterion might be a useful indicator of an effective escape radius for these systems.

Summarizing, as a result of a search performed on a sample of net $\mathrm{H} \alpha$ images of 16 compact groups of galaxies, we have identified 9 star forming tidal objects proposed to be considered dwarf galaxy candidates given their large projected distances from their parent galaxies and their large $\mathrm{H} \alpha$ luminosities. These interesting objects deserve further detailed study, including dynamical and evolutionary aspects which are beyond the scope of the present study.

We acknowledge John Beckman for carefully reading of the last version of this document and for interesting comments and suggestions. Thanks must also be given to A. Zurita for providing HII region catalogues. The INT and the JKT are operated on the island of La Palma by the Isaac Newton Group in the Spanish Observatorio del Roque de Los Muchachos of the Instituto de Astrofísica de Canarias. The $2.2 \mathrm{~m}$ Telescope is operated by the MPIA in the Spanish Observatorio de Calar Alto. This research has made use of the NASA/IPAC Extragalactic Database (NED) which is operated by the Jet Propulsion Laboratory, California Institute of Technology, under contract with the National Aeronautics and Space Administration. This study was partly financed by the Spanish DGES (Dirección General de Enseñanza Superior), grant PB97-0158. 


\section{REFERENCES}

Barnes J.E., 1988, ApJ, 331, 699

Duc P.A., Mirabel I.F., 1997, Messenger, 89, 14

Duc P.A., Mirabel I.F., 1998, A\&A, 333, 813

Elmegreen B.G., Kaufman M., Thomasson M., 1993, ApJ, 412, 90

Ferguson A.M.N., Wyse R.F.G., Gallagher J.S., Hunter D.H., 1998, ApJ, 506, L19

Hibbard J.E., Mihos J.C., 1995, AJ, 110, 140

Hibbard J.E., van Gorkom J.H., 1996, AJ, 111, 655

Hickson P., 1982, ApJ, 255, 382

Hickson P., Kindl E., Auman J.R., 1989, ApJS, 70, 687

Huchtmeier W.K., 1997, A\&A, 325, 473

Hunsberger S.D., Charlton J.C., Zaritsky D., 1998, ApJ, 505, 536

Hunter D.A., Gallagher J.S., 1986, PASP, 98, 5

Iglesias-Páramo J., Vílchez J.M., 1997a, ApJ, 479, 190

Iglesias-Páramo J., Vílchez J.M., 1997b, ApJ, 489, L13

Iglesias-Páramo J., Vílchez J.M., 1998, AJ, 115, 1791

Iglesias-Páramo J., Vílchez J.M., 1999, ApJ, 518, 94

Johnson K.E., Vacca W.D., Leitherer C., Conti P.S., Lipscy S.J., 1999, AJ, 117, 1708

Kennicutt R.C., 1998, ARA\&A, 36, 189

Mendes de Oliveira C., Hickson P., 1994, ApJ, 427, 684

Menon T.K., 1995, MNRAS, 274, 845

Mirabel I.F., Lutz D., Maza J., 1991, A\&A, 243, 367

Mirabel I.F., Dottori H., Lutz D., 1992, A\&A, 256, L19

Moles M., del Olmo A., Perea J., Masegosa J., Márquez I., Costa V., 1994, A\&A, 285, 404

Moles M., Sulentic J.W., Márquez I., 1997, ApJ, 485, L69

Moles M., Márquez I., Sulentic J.W., 1998, A\&A, 334, 473 
Ohyama Y., Nishiura S., Murayama T., Taniguchi Y., 1998, ApJ, 492, L250

Peterson S.D., 1979, ApJS, 40, 527

Rozas M., Beckman J.E., Knapen J.H., 1996, A\&A, 307, 735

Rozas M., Zurita A., Beckman J.E., 2000, A\&A, 354, 823

Rubin V.C., Hunter D.A., Ford W.K., 1990, ApJ, 365, 86

Schweizer F., 1978, in The Structure and Properties of Nearby Galaxies, IAU Symposium No. 77, edited by E. M. Berkhuijsen and R. Wielebinski (Reidel, Dordrecht), p. 279

Terlevich R., Melnick J., 1981, MNRAS, 195, 839

Tomre A., Tomre J., 1972, ApJ, 178, 623

Verdes-Montenegro L., Yun M.S., Perea J., del Olmo A., Ho P.T.P., 1998, ApJ, 497, 89

Verdes-Montenegro L., Yun M.S., Williams B.A., Huchtmeier W.K., del Olmo A., Perea J., 2000, in Small Galaxy Groups, IAU Coll. 174, in press

Vílchez J.M., Iglesias-Páramo J., 1998, ApJS, 508, 248

Williams B.A., Rood H.J., 1987, ApJS, 63, 265

Williams B.A., McMahon P.M., van Gorkom J.H., 1991, AJ, 101, 1957

Xu C., Sulentic J.W., Tuffs R., 1999, ApJ, 512, 178

Zwicky F., 1956, Ergebnisse der Exakten Naturwissenschaften, 29, 344 
Fig. 1.- Greyscale map of HCG 31 in the red continuum band. Overimpossed contours correspond to the net $\mathrm{H} \alpha$ emission. Tidal objects are marked with arrows. Axis units correspond to arcseconds. North is Up and East is Left.

Fig. 2.- Same as Figure 1 for HCG 44.

Fig. 3.- Same as Figure 1 for HCG 92.

Fig. 4.- Same as Figure [1 for HCG 93.

Fig. 5.- Same as Figure 1 for HCG 95. 


\begin{tabular}{|c|c|c|c|c|c|c|}
\hline Name & Parent Gal. & $\begin{array}{l}R_{25} \\
k p c\end{array}$ & $\begin{array}{l}L(\mathrm{H} \alpha) \\
e^{-1 g ~ s^{-1}}\end{array}$ & $x(\%)$ & $\begin{array}{c}E . W .(\mathrm{H} \alpha) \\
\AA\end{array}$ & $\overline{D_{\text {proj }} / R_{25}}$ \\
\hline TD 31a & HCG 31a, HCG 31c & 6.74 & 39.82 & 0.8 & 103 & 1.43 \\
\hline TD 31b & & & 39.96 & 1.1 & 50 & 1.03 \\
\hline TD 31c & & & 40.47 & 3.6 & 219 & 0.98 \\
\hline TD 31d & & & 39.21 & 0.2 & - & 1.37 \\
\hline TD 31e & & & 38.91 & 0.1 & - & 1.53 \\
\hline TD 31f & & & 38.91 & 0.1 & - & 1.69 \\
\hline TD $31 \mathrm{~g}$ & & & 40.44 & 3.4 & 623 & 2.21 \\
\hline TD $31 \mathrm{~h}$ & & & 40.06 & 1.4 & 592 & 2.52 \\
\hline TD 31i & & & 39.21 & 0.2 & - & 3.07 \\
\hline TD 44a & HCG 44d & 3.84 & 39.31 & 3.5 & 188 & 2.33 \\
\hline TD 44b & & & 39.35 & 3.8 & 163 & 1.59 \\
\hline TD $44 \mathrm{c}$ & & & 38.82 & 1.1 & 76 & 1.40 \\
\hline TD 44d & & & 38.68 & 0.8 & 105 & 1.14 \\
\hline TD $44 \mathrm{e}$ & & & 38.73 & 0.9 & 81 & 0.99 \\
\hline TD 44f & & & 38.25 & 0.3 & 118 & 0.97 \\
\hline TD $44 \mathrm{~g}$ & & & 38.38 & 0.4 & 150 & 1.33 \\
\hline TD $44 \mathrm{~h}$ & & & 38.08 & 0.2 & - & 1.22 \\
\hline TD 92a & HCG 92c & 19.51 & 40.36 & 2.8 & 90 & 2.41 \\
\hline TD $92 b$ & HCG 92b, HCG 92d & 19.52 & 41.32 & 1.3 & $\overline{110}$ & 1.14 \\
\hline TD 92c & & & 41.08 & 0.7 & 95 & 1.64 \\
\hline TD 92d & & & 40.71 & 0.3 & - & 0.53 \\
\hline TD 92e & & & 41.11 & 0.8 & 30 & 0.36 \\
\hline TD 92f & & & 40.96 & 0.6 & 33 & 0.45 \\
\hline TD $92 \mathrm{~g}$ & & & 40.74 & 0.3 & - & 1.07 \\
\hline TD 93a & HCG 93b & 10.90 & 39.06 & 0.3 & 23 & 3.02 \\
\hline TD 93b & & & 40.16 & 3.2 & 58 & 2.01 \\
\hline TD 93c & & & 39.39 & 0.6 & 12 & 1.78 \\
\hline TD 93d & & & 39.31 & 0.5 & 16 & 1.30 \\
\hline TD 93e & & & 40.75 & 5.0 & 22 & 0.94 \\
\hline TD 93f & & & 39.65 & 1.0 & 14 & 0.79 \\
\hline TD 93g & & & 39.48 & 0.7 & 12 & 1.07 \\
\hline TD 93h & & & 39.38 & 0.5 & 13 & 1.08 \\
\hline TD 95a & HCG 95c & 7.54 & 40.25 & 7.9 & 23 & 2.66 \\
\hline TD 95b & & & 40.91 & 36 & 179 & 2.02 \\
\hline TD 95c & & & 40.56 & 16 & 20 & 1.34 \\
\hline TD 95d & & & 40.20 & 7.2 & 14 & 0.51 \\
\hline
\end{tabular}

Table 1: Physical properties of the tidal dwarf objects: (1) Name of the dwarfs; (2) Name of the parent galaxy according to Hickson et al. (1989); (3) Radius in $k p c$ of the $\mu_{B}=25 \mathrm{mag} \mathrm{arcsec}^{-2}$ isophote corresponding to the parent galaxy; (4) $\mathrm{H} \alpha$ luminosity in $\mathrm{erg} \mathrm{s}^{-1}$; (5) Fraction of the total $\mathrm{H} \alpha$ luminosity emitted by the dwarf galaxy; (6) H $\alpha$ equivalent width of the dwarf galaxies, expressed in $\AA$; (7) Projected distance to the center of the parent galaxy, in units of $R_{25}$. 
This figure "f1.gif" is available in "gif" format from: http://arxiv.org/ps/astro-ph/0011115v1 
This figure "f2.gif" is available in "gif" format from: http://arxiv.org/ps/astro-ph/0011115v1 
This figure "f3.gif" is available in "gif" format from: http://arxiv.org/ps/astro-ph/0011115v1 
This figure "f4.gif" is available in "gif" format from: http://arxiv.org/ps/astro-ph/0011115v1 
This figure "f5.gif" is available in "gif" format from: http://arxiv.org/ps/astro-ph/0011115v1 